\title{
Correlation of Imageological Findings with Per-Op Findings in Microvascular Decompression for Trigeminal Neuralgia
}

\author{
Authors \\ Dr Pushkraj Baswant Birajdar ${ }^{1}$, Dr Rakesh H. Jasani ${ }^{2}$ \\ ${ }^{1}$ DNB Neurosurgery, Consultant Neurosurgeon, Government Medical College, Latur-413512, Maharashtra \\ ${ }^{2}$ DNB Neurosurgery, Consultant Neurosurgeon, Care Hospital, Hyderabad-01 \\ Email: djdrrakesh@gmail.com \\ Corresponding Author
}

Dr Pushkraj Baswant Birajdar

C/O B.S.Birajdar, Sakar House, Near Jaikranti College, Sitaramnagar,

Latur-413512, Maharashtra, India

Phone-8698312329/8978622653, Email: pushkrajb@gmail.com

\begin{abstract}
Trigeminal neuralgia (TGN) is characterized by sharp, electric shock like paroxysmal lancinating pain in the distribution of one or more branches of the trigeminal nerve on one side. Also characterized by periods of remission and initial response to Carbamazepine. 80 to $90 \%$ cases are caused by compression of the trigeminal nerve at the root entry zone by the superior cerebellar artery. $75 \%$ of patients will ultimately fail medical therapy require a surgical procedure mainly Microvascular Decompression. There is tendency for spontaneous remission with pain free intervals of weeks or months being characteristic, regardless of treatment. Most of patients are evaluated by MRI Brain CISS Scan 1.5 T study before surgery.so this study involves correlation of imaging findings of TGN with per-op findings to predict pre-op surgical planning, approach, outcome and prognosis.
\end{abstract}

Keywords: Trigeminal Neuralgia, Carbamazepine, MRI Brain CISS Scan, Microvascular Decompression.

\section{Introduction}

A. Definition: Trigeminal Neuralgia(TGN) defined as a sudden, severe, usually, unilateral, brief, stabbing, recurrent episodes of pain in the distribution of one or more branches of the Vth nerve with areas of trigger-zone, one of the most painful neuropathic pains with impairment of daily functionality, reduced quality of life and depression

\section{B. Sweet diagnostic Criteria}

A-Pain is paroxysmal, intense, sharp superficial and stabbing type
B - Pain may be provoked from trigger zones or by trigger factors

C - Affecting one or more divisions of the Vth nerve.

D - Pain is unilateral

E - No evident sensory deficits

C. Etiology: The most common cause of idiopathic trigeminal neuralgia is tortuous arteries in pre pontine space:

- Most commonly by Superior Cerebellar Artery (SCA) and Anterior Inferior Cerebellar Artery (AICA). 
- Pontine branches of Basilar artery or rarely by Posterior Inferior Cerebellar Artery or veins.

D. Diagnosis: It is based mainly on clinical history supplemented by physical examination of $\mathrm{CN} \mathrm{V}$, VII and VIII and detailed intra oral examination to rule out dental pain

Compression of nerve root usually when

$$
\begin{aligned}
& >\text { Pain@V2 / V3, compression is } \rightarrow \text { by SCA } \\
& >\text { Pain@V1 } \rightarrow \text { by AICA }
\end{aligned}
$$

Because the clinical findings do not often allow accurate lesion localization, MR imaging are required to visualizing the course of the Vth nerve and in detecting lesions.

High-Resolution Magnetic Resonance Imaging (HR-MRI) with special sequences: CISS (Constructive Interference In Steady State) is used in preoperative evaluation of neurovascular compression in patients with TGN.

\section{E. Treatment}

- A preoperative determination of Microvascular compression in terms of exact localization of the compressing vessel, direction of the vessel and relationship between root entry zone (REZ) and vascularture could be of great value for the neurosurgeon.

- Microvascular Decompression of the nerve root produces a rapid and long-term relief of symptoms in most patients (80-90\%) with vessel-associated TGN.

\section{Aims and Objectives}

- Whether vascular compression in TGN patients could be demonstrated reliably by pre-operative HR-MRI

- How high is the correspondence between MRI results and intraoperative findings.

- This will helps surgeons to properly select the patient and to plan surgery accordingly.

\section{Material and Methods \\ Inclusion Criteria}

- Our study consists of 40 patients examined during Apr-2014 to May- 2016.
- All patients presented with trigeminal neuralgia initially underwent HR-MRI with CISS sequence in radiology dept. and then underwent Microvascular decompression in neurosurgery dept.

- Study consisted of patients with mean age 48 ranging from youngest patient at 30 to oldest at 70 .

- F:M ratio was 1.2:1

\section{Exclusion Criteria}

- Age $<15$ and $>70$ years

- Secondary trigeminal neuralgia

- Imageological protocol:

All patients has been evaluated with $1.5 \mathrm{~T}$ MRI Brain

- T1 axial MRI

- T2 axial MRI

- CISS SCAN (Constructive Interference In Steady State)

- MRI Angiography (MRA)

During imageological examination we determine the type of vascular compression, direction of compression, site of compression, particular vessel causing compression, any abnormality of the vessel causing compression, compression by both artery and vein for trigeminal neuralgia.

\section{Operative protocol}

All patients with primary trigeminal neuralgia underwent Microvascular decompression (MVD), during operation we confirm all the findings which we mentioned in imageological protocol.

$>$ Type of compression

$>$ Direction of compression

$>$ Site of compression

$>$ Particular abnormality of vessel causing compression

Compression by both artery and vein

\section{Intra-Op Findings Assessment}

In patients with trigeminal neuralgia one can identify:

$>$ Presence of neurovascular compression $(\mathrm{NVC})$ 
Degree of NVC

$>$ Nature of the compressing vessel

$>$ Location of NVC along the nerve

$>$ Findings can be confirmed during MVD

\section{Pre-op Imaging Parameters checked}

$>$ Type of compression - which included, probable arterial branch causing compression, and loop of vessel present or not.

> Site of compression - compression at REZ or at cisternal segment etc

$>$ Direction of compression - super medially, superiorly, medially etc.

$>$ Particular abnormality of vessel causing compression

$>$ Thinning of nerve

$>$ Loss of CSF interface

$>$ Compression by both artery and vein

\section{Results}

In 37 of 40 patients - (92.5\%) HR-MRI demonstrated the neurovascular compression in good agreement with the intra operative findings.

In one patient MRI demonstrated the trigeminal nerve compression by superior cerebellar artery while per-operative finding suggested the compression by anterior inferior cerebellar artery.

a)

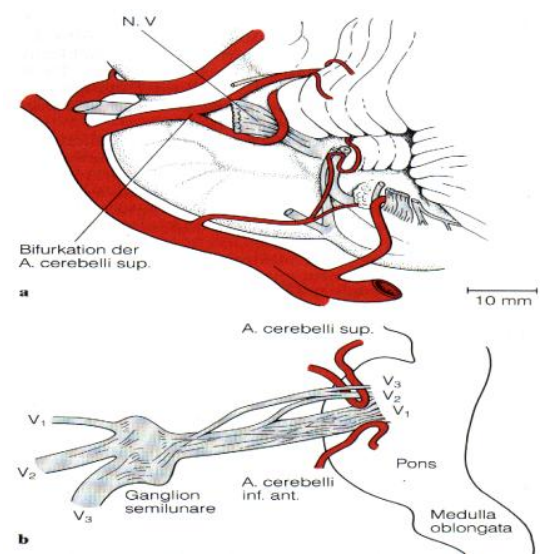

Fig. a: Trigeminal Nerve compression at REZ by SCA/AICA b)

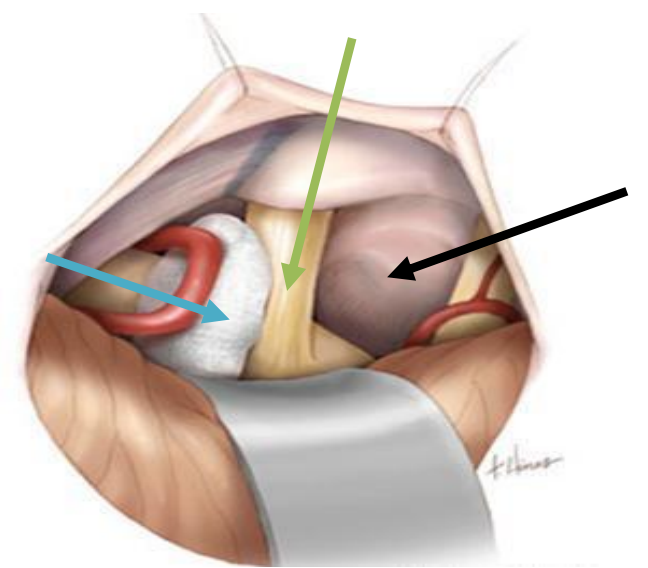

Fig.b: Microvascular Decompression of Trigeminal Nerve

Blue arrow: SCA loop compression

Green arrow: Teflon patch (MVD)

Black arrow: Trigeminal Nerve

\section{c) Branches involvements}

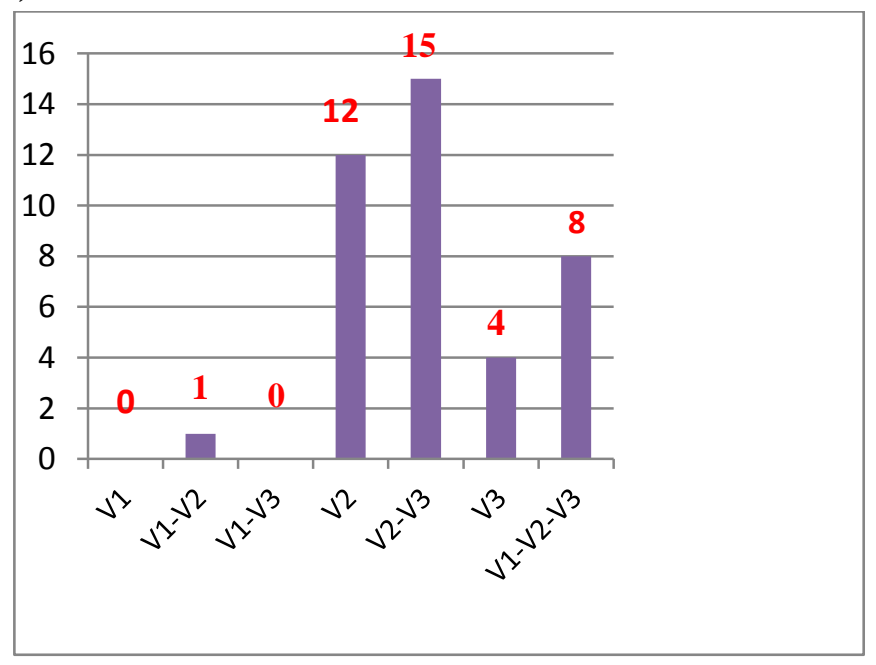

\section{d)Vascular Compression}

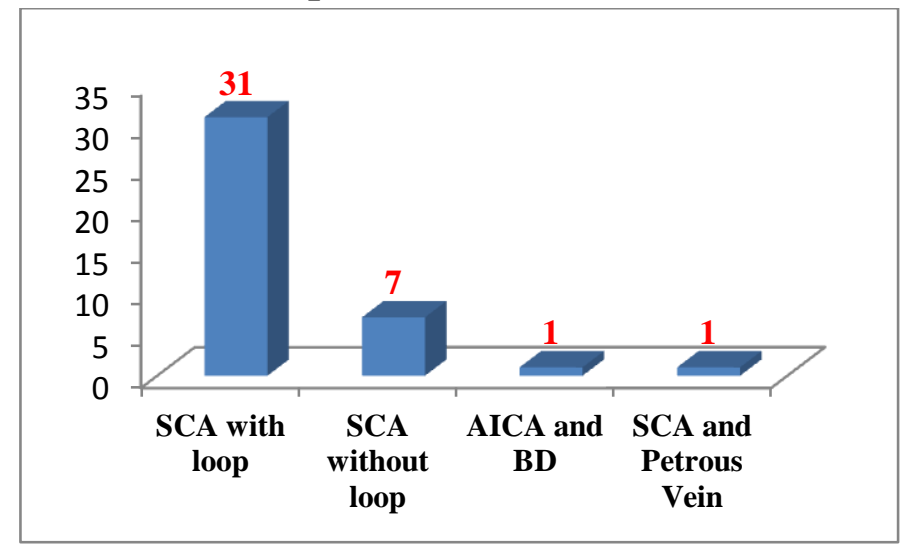




\section{e) Trigeminal Nerve Compression Location}

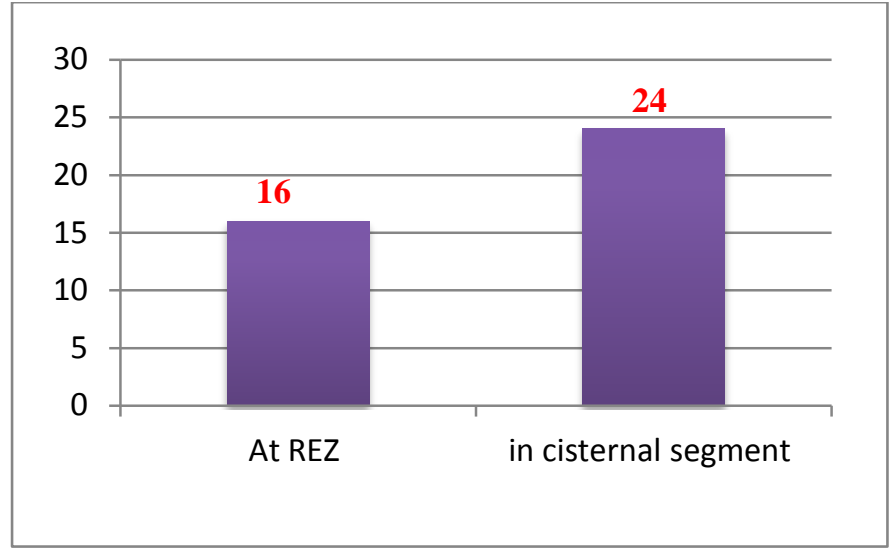

\section{f) Direction Of Compression}

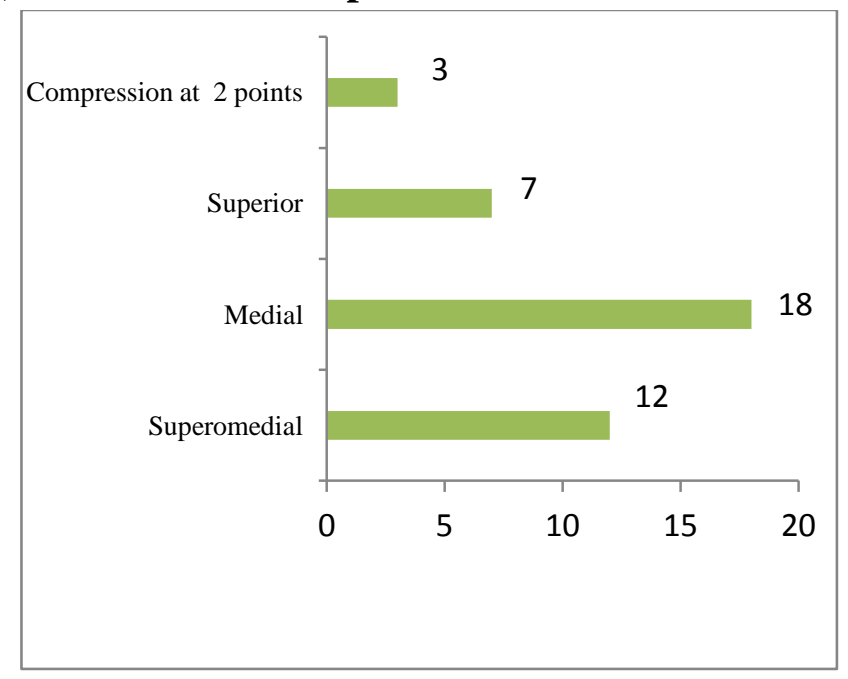

g) MRI and Intraop comparison

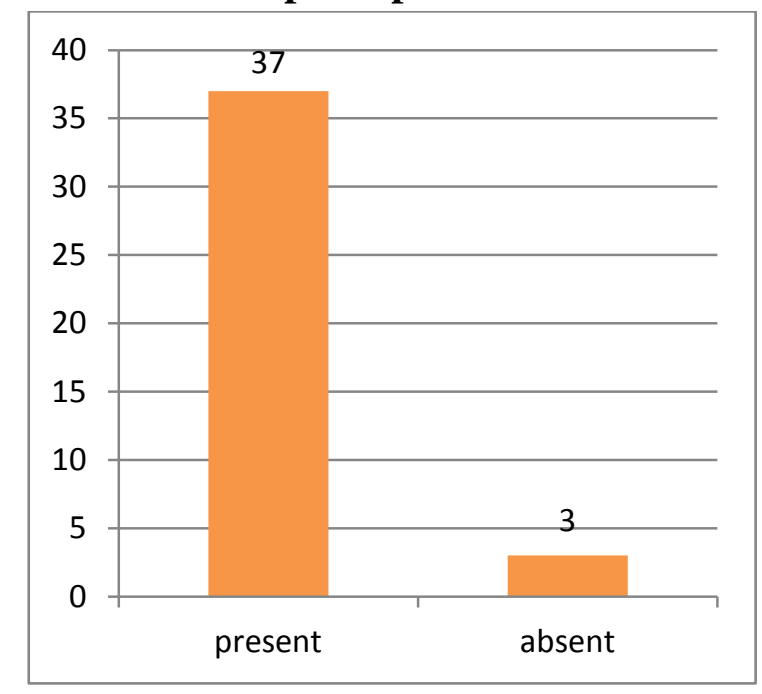

h) MRI CISS scan Brain Image

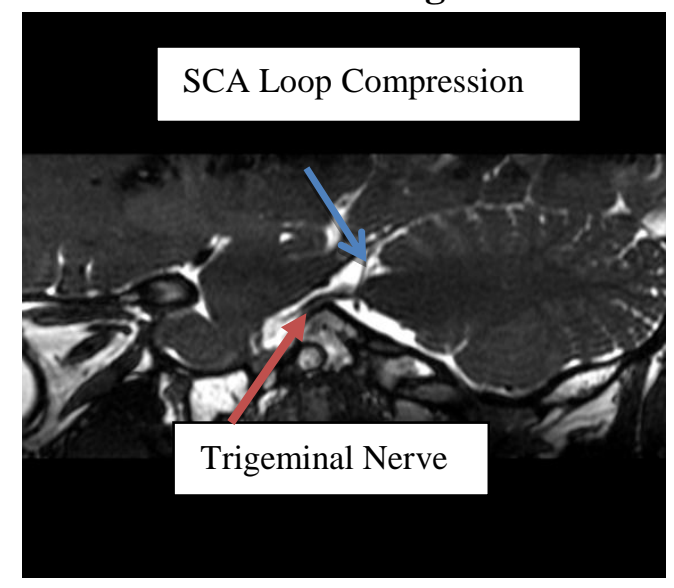

i) SCA loop compressing Vth Nerve

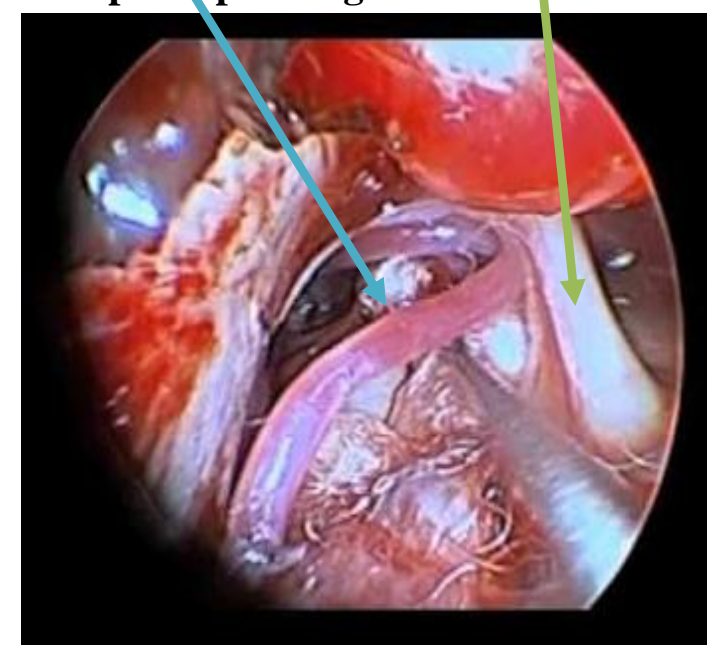

\section{Conclusion}

Our results show that HR-MRI can be used as a decision-making tool for routine preoperative evaluation with high quality MR images.

In patients with clinical presentation of trigeminal neuralgia, we should perform the high resolution MRI, MRA and CISS sequences to overview NVC the trigeminal nerve.

It will help to improve improve patient selection, preoperative preparations for operative planning and intra operative expectations.

\section{Acknowledgement}

I would like to express the deepest appreciation to my teacher Dr.Syed Ameer Basha Paspala, Senior Consultant Neurosurgeon; the Department of Neuro Surgery, CARE Institute of Medical Sciences, Hyderabad who has the attitude and the substance of a genius. He has continuously and convincingly 
conveyed a spirit of knowledge to the research and teaching. Without his guidance and persistent help, this thesis would not have been possible.

I would like to place my thanks on record to Dr.Rakesh H.Jasani,Dr.Rajiv Contractor and for everybody, including the staff of CARE Hospitals, who were important for the successful realization of this thesis. I owe my thanks to my family for providing constant support and encouragement. Last but not the least I thank all my patients who in their periods of sorrow and sufferings have made my study possible with their kind cooperation.

Collective and individual acknowledgements are also owed to my colleagues in the Department of Neuro Surgery and other Departments, whose presence is perpetually refreshing, helpful and memorable.

\section{References}

1. Devor M., Amir R., Rappaport Z.H.: Pathophysiology of trigeminal neuralgia: the ignition hypothesis, Clin.J.Pain2002a; 18 (1): 4-13.

2. Devor M., Govrin-Lippmann R.,Rappaport Z.H.: Mechanism of trigeminal neuralgia: an ultrastructural analysis of trigeminal root specimens obtained during Microvascular decompression surgery, J.Neurosurg. 2002b; 96 (3): 532-543.

3. Jawahar A., Bissonette D.J.,Lunsford L.D.:Imaging the trigeminal nerve and pons before and after surgical intervention for trigeminal neuralgia, Neurosurgery 2001; 48 (1):101-106.

4. Majoie C.B., van Beek E.J.,Valk J., Bosch D.A.: Symptoms and signs related to the trigeminal nerve: diagnostic yield of MR imaging, Radiology 1998; 209 (2): 557-562.

5. Jannetta P.J.: Arterial compression of the trigeminal nerve at the pons in patients with trigeminal neuralgia, J.Neurosurg. 1967; 26(1): Suppl-62

6. Klun B.: Microvascular decompression and partial sensory rhizotomy in the treatment of trigeminal neuralgia: personal experience with 220 patients, Neurosurgery 1992; 30 (1): 49-52.

7. E Lang et al: neurovascular relationship at the trigeminal root entry zone in persistent idiopathic facial pain: finding from MRI 3D visualization(12 patients):JNeurol Neurosurg Psychiatry, 2005;76:1506-1509.

8. Leal PR et al.: visualization of vascular compression of trigeminal nerve with high resolution 3T MRI: prospective study comparing preoperative imaging analysis to surgical findings in 40 consecutive patients who underwent MVD for trigeminal neurallgia: Neurosurgery 2011Jul;69(1):15-25.

9. Bulent Tucer et al.: Microvascular decompression for primary trigeminal neuralgia: $\mathrm{J}$ Korean Neurosurg Soc: 52; 42-47, 2012.

10. Mumtaz Ali et al,: significance of trigger point in trigeminal neuralgia: JPMI 2007 vol 21 No. 03:183-186. 